\title{
Update and critical appraisal of sevelamer in the management of chronic renal failure
}

\author{
This article was published in the following Dove Press journal: \\ Open Access Journal of Urology \\ I September 2010 \\ Number of times this article has been viewed
}

\author{
Jacob Grinfeld \\ Akimichi Inaba \\ Alastair J Hutchison \\ Manchester Institute of Nephrology \\ and Transplantation, The Royal \\ Infirmary, Manchester, UK
}

\begin{abstract}
Sevelamer (Renagel and Renvela), is an orally administered weakly basic anion exchange resin that binds dietary phosphate in the gastrointestinal tract, and is approved for use in the US, Europe and many other countries for the treatment of hyperphosphatemia in adult patients on hemodialysis or peritoneal dialysis. Clinical evidence shows that sevelamer is at least as effective as calcium-based oral phosphate binders in controlling serum phosphate, but with a lower incidence of hypercalcemia. Whilst sevelamer hydrochloride is associated with mild acidosis, sevelamer carbonate does not have this drawback. Use of sevelamer and avoidance of calcium-based binders may slow the progression of vascular calcification in hemodialysis patients, and it also reduces serum low-density lipoprotein-cholesterol levels. There was no between-group difference in all-cause mortality between sevelamer and calcium-based phosphate binder therapy in the primary efficacy analysis of the large $(n>2100)$, 3-year DCOR trial. In the smaller $(n=109)$ nonblind RIND trial in patients new to hemodialysis, data suggest there may be an overall survival benefit with sevelamer versus calcium-based phosphate binder treatment but the evidence on the efficacy of sevelamer in reducing mortality and hospitalization is not strong. The balance of evidence, however, does not strongly support the use of sevelamer over the much less costly calcium-based binders except in patients at risk of hypercalcemic episodes. Further research into cardiovascular and all-cause mortality over a longer time period would be needed to settle this issue, and the relative survival benefits and cost effectiveness of all phosphate binder therapies remains to be fully determined. Despite the relative paucity of data available, sevelamer has established itself as the most widely used binder in the United States and the most widely used noncalcium-based binder worldwide. However, affordability is a major issue for most health economies and in the light of recent economic events is likely to become more prominent.
\end{abstract}

Keywords: sevelamer, phosphate, phosphorus, binder, dialysis, vascular calcification

\section{Introduction \\ Clinical significance of hyperphosphatemia in chronic kidney disease}

Kidney plays a pivotal role in maintaining the homeostasis of serum phosphate and calcium levels. It is the site of synthesis of $1 \alpha$-hydroxylase, which is stimulated by hyperphosphatemia to enable $1 \alpha$-hydroxylation of 25 -hydroxycholecalciferol to 1,25-dihydroxycholecalciferol, the most active form of vitamin D. In progressive chronic kidney disease (CKD), there is increasing impairment of phosphate excretion, elevation of fibroblast growth factor 23 , and reduced production of $1 \alpha$-hydroxylase, resulting in both hyperphosphatemia and hypocalcemia. In the absence of active 
management, these changes result in worsening secondary hyperparathyroidism. Although increasing levels of parathyroid hormone (PTH) may initially facilitate the correction of these metabolic abnormalities (via the stimulation of $1 \alpha$-hydroxylase and inhibition of phosphate resorption), the compensatory mechanisms are gradually overcome as renal function deteriorates. This can result in alterations to the intrinsic "set point" of the parathyroid gland, leading to worsening hyperparathyroidism and, eventually, to autonomous tertiary hyperparathyroidism. ${ }^{1}$

These processes and their sequelae are collectively referred to as "CKD mineral and bone disorder" (MBD)., ${ }^{2,3}$ The clinical spectrum of CKD-MBD ranges from high bone turnover osteitis fibrosa (secondary to high PTH levels) to low bone turnover adynamic bone lesion. Either ends of this spectrum may manifest as bone and joint pain, stiffness, muscle weakness, and increased fracture rate, and is associated with extra-osseous deposition of calcium and phosphate in a variety of tissues.

The most common and clinically significant form of extraosseous calcification seen in patients with end-stage renal disease (ESRD) is vascular calcification. ${ }^{4}$ This is an active process, which occurs in both the intima and the media of arteries, and closely resembles osteogenesis in many ways. Vascular smooth muscle cells, in response to high phosphate levels and probably other stimuli, acquire an osteoblast-like phenotype expressing alkaline phosphatase and laying down a collagen-rich extracellular matrix containing calcium-binding proteins such as osteopontin. High levels of PTH, low-density lipoprotein (LDL) cholesterol, inflammatory markers (such as tumor necrosis factor- $\alpha$ ), advanced glycation end products and low levels of calcification inhibitors (fetuin-A and matrix Gla protein) have all been linked to the progression of vascular calcification or "ossification" as it is sometimes called.

Calcified arteries have been demonstrated to be stiffer (as measured by aortic pulse wave velocity), and this is considered to lead to increases in blood pressure and left ventricular afterload, resulting in left ventricular hypertrophy. ${ }^{5,6}$ Epidemiological studies have demonstrated a statistical link between the incidence of vascular calcification and the incidence of ischemic heart disease, and there is a strong statistical association between vascular calcification and cardiovascular and all-cause mortality in end-stage renal failure patients. ${ }^{7}$

\section{Strategies for managing CKD-MBD}

The main target for therapy in CKD-MBD is hyperphosphatemia. Serum phosphate can be reduced by dietary phosphate restriction, but this is usually not feasible in isolation without promoting protein malnutrition. Standard dialysis (4 hours thrice weekly) removes phosphate from the circulation but in amounts insufficient to effectively control serum levels, PTH secretion, or renal bone disease. It is therefore necessary to reduce significantly the intestinal absorption.

In patients with ESRD, $60 \%-70 \%$ of intestinal phosphate is absorbed by 2 processes: (1) passive diffusion across an electrochemical gradient between cells and (2) a transcellular $\mathrm{Na}^{+}$dependent pathway via a cotransporter. ${ }^{8}$ The former plays a far greater role when the intraluminal phosphate concentration increases after a meal. Approximately 60\%-70\% phosphate is absorbed in the duodenum and jejunum and $30 \%-40 \%$ in the ileum. It is not absorbed in the stomach. Potentially, phosphate may be bound in any part of the small bowel and stomach. In order to facilitate minimum phosphate absorption, binding in the stomach is ideal before it reaches the jejunum. There are several phosphate binders that are effective in this role: aluminium-based salts, calcium-based salts, and the more recently introduced sevelamer hydrochloride and lanthanum carbonate. ${ }^{9}$ Other binders that are currently under development include magnesium iron hydroxycarbonate and colestilan. These binders are taken up with meals and bind intraluminal phosphate, preventing its absorption.

Aluminium-based phosphate binders were the first to be generally utilized in the $1970 \mathrm{~s}$. As aluminium is mainly excreted via the kidneys, accumulation was common in patients with stage 5 CKD. However, toxicity arose primarily from aluminium-contaminated dialysis water. Aluminium accumulated predominantly in the central nervous system, bone, and hematopoietic cells causing encephalopathy, osteomalacia, and microcytic anemia, respectively. ${ }^{10,11}$ In certain selected circumstances, aluminium-based phosphate binders may still be used, but this is becoming increasingly uncommon. In the 1990s, calcium carbonate and calcium acetate replaced aluminium-based salts. Since phosphate binding is $\mathrm{pH}$ dependent, high doses are often required, especially if taken with drugs that increase gastric $\mathrm{pH}$. Hypercalcemia is common and occurs approximately in one-third of patients using calcium carbonate. ${ }^{12}$ Calcium intake may correlate with arterial calcification and, therefore, contribute to the increased risk of cardiovascular mortality in ESRD patients, although this view is controversial with no robust data to support it as yet. ${ }^{13}$

Sevelamer is an alternative oral phosphate binder, which contains neither calcium nor aluminium, and is a weakly basic anion-exchange resin. It is a polymer of cross-linked polyallylamine that is not absorbed from the gut and that binds phosphate in exchange for chloride, most optimally 


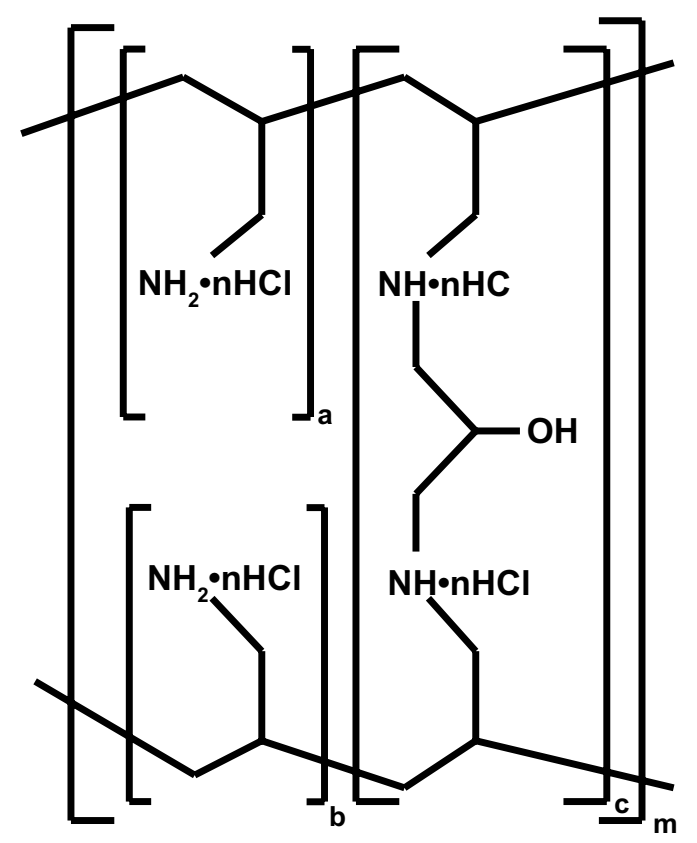

Hydrochloride

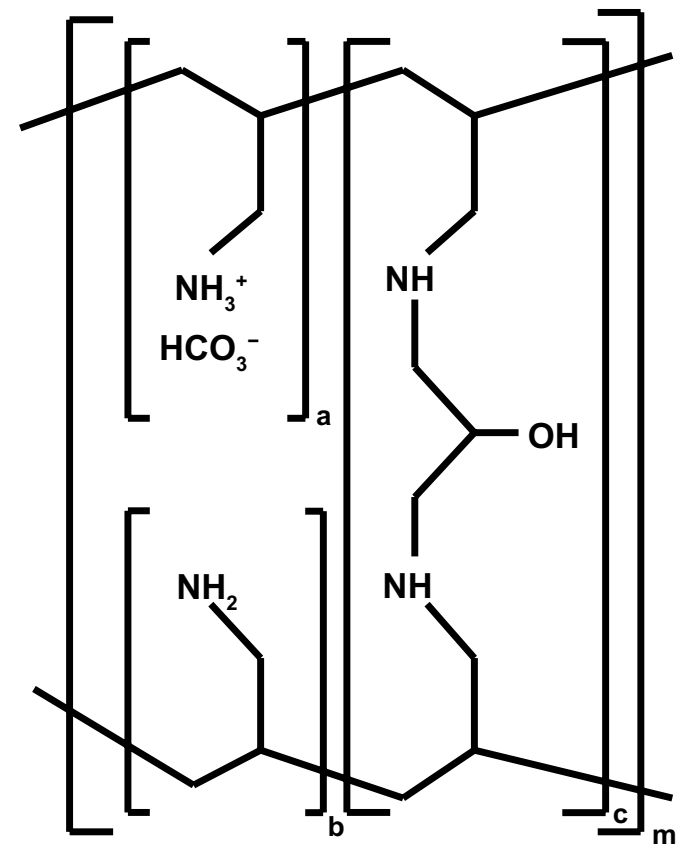

(Bi)carbonate

Figure I Chemical structure of sevelamer hydrochloride and carbonate.

Notes: $a$ and $b$, number of primary amine groups $(a+b=9)$; , number of crosslinking groups $(c=1)$; $n$, fraction of protonated amines (hydrochloride) $(n=0.4)$; $m$, large number to indicate extended polymer network. The primary amine groups shown in the structure are derived directly from poly(allylamine hydrochloride). The cross-linking groups consist of 2 secondary amine groups derived from poly(allylamine hydrochloride) and I molecule of epichlorohydrin. ${ }^{21}$

at $\mathrm{pH} 7 .{ }^{14}$ It was the first nonaluminium, noncalcium-based binder available in the United States and Europe and was initially produced and marketed as the hydrochloride (Renagel ${ }^{\circledR}$, Genzyme Corporation, Cambridge, MA, USA). However, Renagel is associated with exacerbation of acidosis in some patients; therefore, in 2009 production was switched to the carbonate (Renvela, Genzyme Corporation, Cambridge, MA, USA). ${ }^{15}$ This review examines the evidence for the use of sevelamer in the management of CKD, particularly in comparison to calcium-based binders.

\section{Pharmacology of sevelamer}

\section{Structure and binding mechanism}

Sevelamer is a cationic hydrogel, comprising polyallylamine chloride cross-linked with epichlorohydrin (Figure 1), a structure that makes it hydrophilic but insoluble in water. It has a molecular weight of $10^{16} \mathrm{Da} .{ }^{16}$ About $40 \%$ of the multiple amine groups, each separated by 1 carbon on the polymer backbone, become protonated in the gastrointestinal tract and bind the negatively charged phosphate anions, predominantly via ionic and hydrogen bonding. ${ }^{17,18}$ Wrong and Harland ${ }^{19}$ have previously published a detailed description of the structure and properties of sevelamer and other anion-exchange resins in "Nephron" for those who wish to read an in-depth review. ${ }^{19}$
Peak binding occurs in vitro in the physiological $\mathrm{pH}$ range (approximately 7) and is significantly affected by changes in gastric acidity. An in vitro study demonstrated that at neutral $\mathrm{pH}$, sevelamer bound $2.6 \mathrm{mmol} / \mathrm{L}$ of phosphate (at an estimated physiological concentration of $5 \mathrm{mmol} / \mathrm{L}$ ) per gram of binder. Saturation binder capacity was estimated to be about $7 \mathrm{mmol} / \mathrm{g}$ at phosphate concentrations $>50 \mathrm{mmol} / \mathrm{L}$. Sevelamer molecules are too large to be absorbed from the gastrointestinal tract, and the particles become hydrated in gastric and intestinal fluids, swelling more than 6 times their weight in vitro..$^{20,21}$ It is a nonselective anion exchanger, and as a consequence it faces the risk of competitive binding to other anions, and thereby displacement of phosphate, as it progresses through the intestine. By this mechanism, sevelamer hydrochloride binds bile acids and reduces serum levels of LDL cholesterol but at the expense of previously bound phosphate ions that are released. It may also bind lipophilic drugs, such as immunosuppressants, and the fat soluble vitamins D, E, and K. Reduction of 25-hydroxyvitamin D has been reported in a 12-month study. ${ }^{22}$

\section{Drug interactions}

Burke et al ${ }^{17,23}$ conducted studies funded by the Genzyme corporation into drug interactions between sevelamer hydrochloride 
and four commonly used medications: enalapril, metoprolol, digoxin, and warfarin. Four separate open-label, randomized, cross-over experiments were conducted involving between 14 and 32 subjects. Serum measurements of the respective drugs were taken at regular intervals for up to 144 hours following simultaneous administration. No significant differences were noted in serum concentrations of the drugs. Although methodologically rigorous, this series of studies was of short duration and comprised healthy subjects who do not adequately reflect the renal patient group with multiple morbidities. A small, randomized, cross-over study involving 15 subjects reported a $48 \%$ reduction in the bioavailability of oral ciprofloxacin when concomitantly administered with sevelamer. ${ }^{24}$

\section{Altered metabolism of immunosuppressants}

Given that sevelamer may be helpful in patients with failing renal transplants, which provide diminishing phosphate control, a number of papers have examined sevelamer hydrochloride's interaction with immunosuppressants, such as cyclosporin A and mycophenolate mofetil (MMF). Sevelamer had little effect on cyclosporin A levels, ${ }^{25}$ but further studies looking at the metabolites of ciclosporin A have demonstrated that the area under the curve and peak concentration of only one of the metabolites, AM1, were significantly reduced by approximately $30 \%$ and $25 \%$, respectively, 4 days after commencing sevelamer. Other metabolites, such as AM9 and AM4N, remained unchanged. Larger studies are necessary to explore both sevelamer's interactions over a longer period and sevelamer's interactions with more than 30 known metabolites of cyclosporin A.

Concomitant administration of sevelamer hydrochloride and MMF significantly reduced the immunosuppressant's area under the curve by $25 \%$ even after a single dose. ${ }^{25}$ While this drug interaction was demonstrated in a small study involving only 18 subjects over a 4-day period, the results suggest the need for either dose augmentation of MMF or staggered administration of MMF.

A single case report describes a potential interaction of sevelamer hydrochloride and tacrolimus. ${ }^{26}$ A 55-year-old male transplant patient was commenced on sevelamer for phosphate control and subsequently developed subtherapeutic levels of tacrolimus. While taking sevelamer, the patient's peak level of tacrolimus was found to be $9.9 \mathrm{ng} / \mathrm{mL}$ and the area under the curve was found to be $4.02 \mathrm{ng} / \mathrm{h} / \mathrm{mL}$. After stopping sevelamer, the patient's peak and area under the curve levels of tacrolimus increased significantly to $13.1 \mathrm{ng} / \mathrm{mL}$ and $9.69 \mathrm{ng} / \mathrm{h} / \mathrm{mL}$, respectively. Again, further studies are required to confirm this finding.

\section{Clinical efficacy studies}

\section{Effect on serum calcium and phosphate}

Sevelamer has been shown to cause a smaller increase in serum calcium compared with calcium-based phosphate binders, and as expected given its composition, its use is associated with fewer episodes of hypercalcemia. Several trials have shown sevelamer to effect similar reductions in phosphate and calcium $\times$ phosphate product $(\mathrm{Ca} \times \mathrm{P})$ levels as with calcium-based binders. The Treat-to-Goal study, which randomized 200 hemodialysis patients to either sevelamer or calcium-based phosphate binders, demonstrated a decrease in phosphate of approximately $2.5 \mathrm{mg} / \mathrm{dL}$ and $\mathrm{Ca} \times \mathrm{P}$ of approximately $20 \mathrm{mg}^{2} / \mathrm{dL}^{2}$ in both groups $(P=0.33$ and $P=0.12$, respectively), but an $0.4 \mathrm{mg} / \mathrm{dL}$ increase in serum calcium in the calcium-treated group, compared with $0.1 \mathrm{mg} / \mathrm{dL}$ in the sevelamer group $(P=0.002) .{ }^{27}$ Similar results were seen in a number of subsequently published, smaller, randomized controlled studies. ${ }^{28-31}$ Differences in the incidence of hypercalcemia between calcium-treated and sevelamer-treated patients range from $11 \%$ to $51 \%(P=0.04$ and $P=0.001$, respectively). ${ }^{27,30}$

Superior phosphate control with sevelamer has been reported in some studies. Kokuho et $\mathrm{al}^{32}$ switched 113 Japanese patients from calcium carbonate to sevelamer and observed a significant improvement in levels of serum phosphate $(6.1-5.6 \mathrm{mg} / \mathrm{dL}, P=0.001)$ and $\mathrm{Ca} \times \mathrm{P}\left(56.6-49.5 \mathrm{mg}^{2} / \mathrm{dL}^{2}\right.$, $P<0.001)$ plus a fall in calcium $(9.3-8.9 \mathrm{mg} / \mathrm{dL}, P<0.001)$. These results are difficult to interpret reliably given the lack of a control group. One small, randomized, controlled trial in hemodialysis patients has shown better phosphate control with sevelamer $(6.7-6.2 \mathrm{mg} / \mathrm{dL}, P<0.05)$ compared with calcium carbonate, which resulted in an increase in mean serum phosphate $(6.5-6.7 \mathrm{mg} / \mathrm{dL}, P<0.05) .^{33}$

The BRiC study randomized 101 patients to either sevelamer or calcium acetate and showed similar improvements in phosphate control $(2.3-1.87 \mathrm{mmol} / \mathrm{L}$ for the calcium group vs $2.3-1.71 \mathrm{mmol} / \mathrm{L}$ for sevelamer, not significant) but, in contrast to the other studies mentioned here, found no difference in ionized calcium levels $(1.23-12.7 \mathrm{mmol} / \mathrm{L}$ vs $1.23-1.28 \mathrm{mmol} / \mathrm{L}$, not significant) or in the incidence of hypercalcemic episodes (figures not provided). ${ }^{34}$ This may be because patients were randomized to calcium acetate (which has a lower calcium load compared with calcium carbonate) in the calcium-based binder group, whereas other studies did not differentiate between the two binders, and used calcium carbonate alone, or failed to specify which was used. A retrospective review of 30 patients, which was carried out more than 1 year- longer than the follow-up of 
most of the aforementioned studies-showed significantly higher phosphate (2.1 vs $1.74, P=0.0013)$ in the sevelamertreated group. ${ }^{35}$ These results are difficult to interpret given the retrospective, nonrandomized nature of the trial, and the fact that figures are not given for baseline serum phosphate levels.

More definitive evidence was provided by the Calcium Acetate Renagel Evaluation (CARE) study, in which 128 maintenance hemodialysis patients were randomized to either calcium acetate or sevelamer for 8 weeks. A significantly higher serum calcium $(P<0.0001)$, but lower phosphate $(P=0.0006)$ and $\mathrm{Ca} \times \mathrm{P}(P=0.022)$, was seen in the calciumtreated group. ${ }^{36}$ With the publication of the Kidney Disease: Improving Global Outcomes (KDIGO) bone and mineral metabolism guidelines, the use of $\mathrm{Ca} \times \mathrm{P}$ is no longer felt to be a useful indicator and does not offer additional information beyond individual levels of calcium and phosphate. ${ }^{3}$

\section{Effect on intact PTH and bone metabolism/turnover}

It has been hypothesized that use of calcium may oversuppress intact PTH (iPTH) levels, leading to decreased uptake of calcium by bone with decreased bone turnover and density and increased vascular calcification. ${ }^{37}$ Asmus et $\mathrm{al}^{29}$ randomized patients either to calcium carbonate or to sevelamer and examined both biochemical markers and bone density as measured using electron beam computed tomography (EBCT). Calcium-based binders were found to cause significant iPTH suppression compared with sevelamer $(P<0.001)$, whereas sevelamer-treated patients had higher levels of alkaline phosphatase $(P=0.046)$. Treatment with calcium was associated with a decrease in trabecular bone density at 2 years, whereas sevelamer-treated patients showed a significant increase. Barreto et $\mathrm{al}^{34}$ also reported higher alkaline phosphatase, deoxypyridinoline, and iPTH levels in the sevelamer-treated group but no differences in bone turnover on histological assessment. Similar results were seen for iPTH and bone turnover in a 2008 study of 119 hemodialysis patients but improved trabecular architecture was described in the sevelamer group. ${ }^{31}$ No significant differences in iPTH were reported in the CARE study, which used calcium acetate, or in Russo et al's study of 90 predialysis patients, which used calcium carbonate. ${ }^{36,38}$

\section{Effect on LDL, high-density lipoprotein, and total cholesterol}

The ability of sevelamer to lower serum LDL and total cholesterol levels and to potentially increase high-density lipoprotein (HDL) levels is well documented and is considered to be secondary to its ability to sequester bile acids. This has been demonstrated in hemodialysis patients, with an average decrease of $40 \mathrm{mg} / \mathrm{dL}$ in total cholesterol $(P<0.0001)$ and $38 \mathrm{mg} / \mathrm{dL}$ in LDL cholesterol $(P<0.0001)$ at 52 weeks reported in the Treat-to-Goal study. ${ }^{27}$ A later, smaller study also demonstrated improvement in total cholesterol and LDL cholesterol, but to a lesser extent while showing a rise in HDL cholesterol of $6.2 \mathrm{mg} / \mathrm{dL}(P=0.03)$. A study of 49 predialysis patients also showed improvements in serum LDL and total cholesterol but no significant change in HDL cholesterol. ${ }^{39}$

\section{Effects on markers of inflammation, endothelial dysfunction, and calcification inhibitors}

A number of circulating factors involved in the regulation of osteogenesis has been implicated in the progression of vascular calcification (discussed later). Among these is fetuin-A, a calcification inhibitor, levels of which are decreased in inflammation and $\mathrm{CKD}$, and which are inversely proportional to the prevalence and severity of vascular calcification, as well as cardiovascular and all-cause mortality. ${ }^{40}$ Caglar et a ${ }^{41}$ randomized 50 patients with stage $4 \mathrm{CKD}$ to either sevelamer or to calcium acetate and demonstrated a significant increase in fetuin-A levels after 8 weeks of treatment with sevelamer but not with calcium $(P<0.001)$. This correlated with an improvement in flow-mediated dilatation, a marker of endothelial dysfunction. Othmane et $\mathrm{al}^{42}$ however, did not observe any differences in fetuin-A nor any other measured calcification inhibitors - matrix GLA protein, osteoprotegerin or soluble receptor activator for nuclear factor $\kappa \mathrm{B}$ ligand. It is worth noting that this study was not controlled at baseline for markers of calcium and phosphate metabolisms. Both Caglar et al and Takei et al demonstrated a decrease from baseline in C-reactive protein levels in sevelamer-treated patients (by $15-10 \mathrm{mg} / \mathrm{L}$, $P<0.001$ and $14-11 \mathrm{mg} / \mathrm{L}, P=0.038$, respectively), suggesting a possible anti-inflammatory role for sevelamer. ${ }^{33,41}$ The mechanism by which this anti-inflammatory effect might occur is not clear, given sevelamer's lack of absorption, and indeed, this finding is not replicated in all studies. One tentative explanation, based on the finding of a pilot study, is that sevelamer binds endotoxin in the intestinal lumen, but no clinical trials have yet investigated this hypothesis. ${ }^{43}$

\section{Effect on vascular and valvular calcification}

Hyperphosphatemia, hypercalcemia, hyperlipidemia, and reduced levels of calcification inhibitors are seen in CKD and have all been implicated in vascular calcification. This in turn is associated with an increase in systolic blood pressure and 
arterial stiffness, as well as an increased incidence of ischemic heart disease and all-cause mortality. Whether calcium-based binders accelerate the process of vascular calcification by creating a positive calcium balance and oversuppressing PTH remains unproven but is a widely held view. ${ }^{44} \mathrm{~A}$ number of studies have examined the progression of vascular calcification in calcium-treated patients compared with sevelamer.

In the Treat-to-Goal Study, the degree of coronary artery, aortic, and valvular calcification was measured by EBCT scan at baseline, 26 and 52 weeks as a secondary outcome measure. Significant progression of aortic artery calcification was seen in the calcium-treated group but not in the sevelamer-treated group ( $P=0.67$ vs $P=0.0002$ at the 52 -week time point), with similar results for coronary artery calcification $(P=0.43$ vs $P=0.0007)$. However, although the results were reported as percentage changes and absolute values, the extent of calcification at baseline was different between the groups. This was criticized on the basis that the more heavily calcified sevelamer group was perhaps unable to progress further. Valvular calcification did not change significantly in either group. Similar results were found in a 2005 study of 109 patients new to hemodialysis. ${ }^{45}$ These studies have also been criticized for their lack of blinding, the fact that patients treated with calcium acetate and carbonate were combined in the same treatment group (despite differences in phosphate-binding efficacy and calcium load), and lack of adequate control of vitamin D dosing, LDL levels, and dialysate calcium. ${ }^{46}$

Braun et $\mathrm{al}^{47}$ studied 114 dialysis patients and found significant increases in aortic and coronary artery calcification in calcium carbonate - treated patients $(P<0.01$ for both), but no significant change in sevelamer-treated patients after 52 weeks. The difference in aortic calcification was even more marked after 2 years of treatment in a follow-up study of a subgroup of these patients. ${ }^{29}$ Takei et al ${ }^{33}$ also compared sevelamer to calcium carbonate, with similar results in terms of aortic calcification in a study of 42 patients. However, no difference was observed after 12 months in the BRiC study of sevelamer vs calcium acetate, in which monthly adjustments were made to calcitriol and dialysate prescriptions on the basis of phosphate, calcium, and iPTH levels in order to avoid PTH oversuppression. ${ }^{34}$ Galassi et al ${ }^{30}$ studied both diabetic and nondiabetic dialysis patients time points of 6,12 , and 18 month but did not differentiate between calcium carbonate and acetate in the calcium treatment arm. Significant differences were only seen in the diabetic patients after 18 months. Interestingly, a small study by Othmane et $\mathrm{al}^{42}$ compared changes in aortic pulse wave velocity in 13 sevelamer-treated hemodialysis patients with matched controls and demonstrated an improvement in vascular stiffness at 11 months $(P=0.042)$. Russo et al ${ }^{38}$ observed a lack of a significant difference in total calcium score after an average of 2 years (415-453, not significant, $P$ value not supplied), in contrast to the rise seen in patients treated with a low-phosphate diet alone $(360-547, P=0.001)$ or with calcium carbonate $(340-473, P=0.001)$.

The CARE-2 study also compared sevelamer with calcium acetate over a 1-year period, but additionally added atorvastatin therapy to reach a cholesterol target of less than $70 \mathrm{mg} / \mathrm{dL}$ in both groups, hypothesizing that the lower rates of vascular calcification seen with sevelamer treatment may be secondary to its effects on lipids. ${ }^{48}$ Similar rates of vascular calcification were seen in both groups. Taken together, these studies suggest sevelamer may have a role in slowing the rate of vascular calcification when compared with calcium carbonate, but possibly not to calcium acetate.

\section{Effect on uric acid levels}

The incidence of hyperuricemia and gout is increased in patients with CKD due to impaired uric acid excretion. The ability of sevelamer to reduce uric acid levels was first demonstrated by Garg et $\mathrm{al}^{49}$ who performed an unblinded, randomized, controlled trial comparing the effects of sevelamer with calcium-based binders on uric acid levels in 200 maintenance hemodialysis patients. After 52 weeks, the sevelamer group was found to have a mean reduction in uric acid levels of $-0.64 \mathrm{mg} / \mathrm{dL}$ compared with $-0.26 \mathrm{mg} / \mathrm{dL}$ in the calcium-treated arm, with a change in uric acid levels proportional to the baseline uric acid levels. They hypothesized that this was secondary to a decrease in production, rather than elimination, given the comparable creatinine levels and efficacy of dialysis between the 2 groups. Ohno et $\mathrm{al}^{50}$ performed an uncontrolled study in which they added sevelamer to 127 maintenance dialysis patients' existing binder treatment and found a $0.44 \mathrm{mg} / \mathrm{dL}$ decrease in urate levels after 6 months in those with baseline hyperuricemia, but no significant difference in those with normal baseline uric acid. On the basis of in vitro studies, they proposed that this is due to a direct urate-binding effect in the gastrointestinal tract.

\section{Safety and tolerability Metabolic acidosis}

Sevelamer hydrochloride has been associated with a number of adverse effects, the most significant of which is its association with metabolic acidosis. A number of theories on the pharmacological mechanisms of acidosis have been posited, the most common being that the withdrawal of other 
phosphate binding agents, which are often alkalinizing agents such as carbonates and acetates, may precipitate a rapid acidosis. ${ }^{51}$ Examining the phosphate binding model, Brezina et $\mathrm{al}^{52}$ also noted that for every phosphate molecule bound to sevelamer, one $\mathrm{HCl}$ molecule is liberated. Furthermore, sevelamer may bind to sodium bicarbonate, secreted by the pancreas into the small intestine, in exchange for chloride. Finally, when sevelamer binds to a molecule of bile acid as a part of its lipid lowering effect, there is net production of one molecule of $\mathrm{HCl}$. Regardless of the exact mechanism, chronic metabolic acidosis adversely affects both morbidity and mortality, and therefore, should be corrected.

With the exception of one earlier study by Chertow et $\mathrm{al}^{53}$ which showed a slight initial increase in sodium bicarbonate concentrations in a 46-week trial, there is general consensus that metabolic acidosis develops in the weeks after commencing sevelamer hydrochloride. ${ }^{54-57}$ However, the chronicity of the acidosis is unclear. Confounding any studies is the concomitant use of sodium bicarbonate supplementation, and underlying deterioration in renal production of bicarbonate. Vlahakos et $\mathrm{al}^{56}$ demonstrated a drop in serum $\mathrm{pH}$ and serum bicarbonate levels with sevelamer introduction, followed by a partial but incomplete recuperation over a 2 -year period. In contrast, the use of calcium carbonate had no effect on these measurements. After 3 months of sevelamer introduction, mean $\mathrm{pH}$ had dropped to a nadir from 7.4 to $7.35(P<0.0001)$ and bicarbonate dropped from approximately $27 \mathrm{mmol} / \mathrm{L}$ to approximately $24 \mathrm{mmol} / \mathrm{L}(P<0.01)$. Supplemental bicarbonate remained constant over the course of the study. Subsequently, some recovery in $\mathrm{pH}$ and sodium bicarbonate was observed.

The recent manufacture and introduction of sevelamer carbonate (Renvela) may have solved the problem of acidosis, and the hydrochloride was removed from the US market in October 2009. ${ }^{15}$ However, Renvela is not currently available in most European countries, and Harland ${ }^{58}$ argues that it is misnamed since it does not contain carbonate.

\section{Commonly observed side effects}

Sevelamer hydrochloride functions exclusively within the gut lumen and, as with all oral phosphate binders, the majority of common side effects are related to gastrointestinal upset. ${ }^{1}$ The Genzyme Corporation's own literature reports that during a 52-week trial of sevelamer in dialysis patients, the most common side effects in descending order were vomiting (22\%), nausea (20\%), diarrhea (19\%), dyspepsia (16\%), abdominal pain $(9 \%)$, flatulence $(8 \%)$, and constipation $(8 \%) .{ }^{22}$ Cases of fecal impaction and, less commonly, ileus, bowel obstruction, and perforation have been reported, possibly related to the large increase in size as the molecule hydrates in the gastrointestinal tract. ${ }^{59,60}$

In 143 peritoneal dialysis patients studied for 12 weeks, most adverse reactions were similar to adverse reactions observed in hemodialysis patients. The most frequently occurring treatment-emergent serious adverse reaction was peritonitis ( 8 reactions in 8 patients [8\%] in the sevelamer group vs 2 reactions in 2 patients [4\%] on active control group). Thirteen patients (14\%) in the sevelamer group and 9 patients $(20 \%)$ in the active control group discontinued, mostly for gastrointestinal adverse reactions. Therefore, Genzyme advises that patients on peritoneal dialysis should be monitored to ensure the reliable use of appropriate aseptic technique with prompt recognition and management of any signs and symptoms associated with peritonitis. ${ }^{22}$

\section{Sevelamer carbonate}

Sevelamer carbonate shares a similar structure to sevelamer hydrochloride (Figure 1). Both sevelamer carbonate and hydrochloride are marketed as $800 \mathrm{mg}$ tablets, but a powdered form of sevelamer carbonate is also available in foil-lined packets containing 800 or $2,400 \mathrm{mg}$. Apart from the absence of induction of metabolic acidosis, sevelamer carbonate appears to be equivalent to the hydrochloride in terms of efficacy and tolerability ${ }^{61}$

\section{Dosing of sevelamer}

The average prescribed dose of sevelamer is $7.2 \mathrm{~g}$ per day, or nine $800 \mathrm{mg}$ pills. ${ }^{22}$ In practice, this means that many patients are asked to take 12 or more sevelamer pills daily. The introduction of a 2.4-g packet of sevelamer carbonate provides the possibility of an average dose of 1 packet 3 times daily. This might be expected to offer significant advantages in terms of adherence to treatment.

\section{Cost comparison with available phosphate binders}

Sevelamer is significantly more expensive than calcium-based binders with price per tablet approximately 10 times that of calcium acetate and 50 times that of calcium carbonate (Table 1). Given the paucity and uncertainties of outcome data reported for studies of sevelamer (and all other phosphate binders), pharmacoeconomic analysis is extremely difficult. However, Taylor et $\mathrm{al}^{62}$ report an attempt to estimate the costs and outcomes associated with sevelamer treatment for hyperphosphatemia compared with calcium-based binders. They concluded that patients receiving sevelamer might be expected to experience 2.70 quality-adjusted life years (QALYs) compared with 2.46 for those treated with calcium carbonate (ie, an incremental 
Table I Relative cost of commonly available oral phosphate binders in the United Kingdom

\begin{tabular}{|c|c|c|c|}
\hline Phosphate binders & $\begin{array}{l}\text { Basic net price } \\
\text { per tablet }^{\mathrm{a}}(\boldsymbol{t})\end{array}$ & $\begin{array}{l}\text { Usual dose range } \\
\text { (g/d) }\end{array}$ & $\begin{array}{l}\text { Annual cost } \\
\text { range }(t)\end{array}$ \\
\hline Aluminium hydroxide (475 mg) & 0.03 & $1.9-5.7$ & $43.80-131.40$ \\
\hline Calcium carbonate ( $1.25 \mathrm{~g})$ & 0.09 & $2.5-7.5$ & $65.70-197.10$ \\
\hline Calcium acetate (I g) & 0.11 & $2.0-6.0$ & $80.30-240.90$ \\
\hline $\begin{array}{l}\text { Sevelamer } \\
\text { hydrochloride }(800 \mathrm{mg})\end{array}$ & 0.66 & $2.4-12.0$ & $722.70-3613.50$ \\
\hline $\begin{array}{l}\text { Lanthanum carbonate } \\
(500,750 \text {, or } 1,000 \mathrm{mg})\end{array}$ & $1.27,1.69,1.80$ & $1.5-3.0$ & $1390.65-197 \mid .00$ \\
\hline
\end{tabular}

Notes: aCosting and usual dose range taken from "British National Formulary 58”, BMJ Group and RPS Publishing 2009, London, United Kingdom. Costs to the NHS are greater than the net prices, which indicate relative cost.

gain of 0.24 QALYs). This would result in an incremental cost per QALY of $£ 27,120$ and an incremental cost per life year gained of $£ 15,508$, which the authors concluded as "a modest investment of additional economic resources". This study utilized a 5-year decision model, based on a probably overoptimistic estimate of a $79 \%$ reduction in the risk of hospitalization for the first 18 months of the model.

\section{Patient focused perspectives}

No randomized trials of sevelamer have reported a measure of health-related quality of life. Neither are there any reports of cardiovascular events nor the frequency of symptomatic bone disease such as fractures or bone pain.

Only 1 study to date has examined all-cause mortality as a primary end point in comparisons of sevelamer with calcium-based phosphate binders. ${ }^{63}$ The Dialysis Clinical Outcomes Revisited (DCOR) trial randomized 2,103 patients to sevelamer or calcium-based binders (unspecified) and followed them for up to 44 months, although only 1,068 completed the trial. Binder type and dosage were not monitored during the study. No differences were found for all-cause or cardiovascular deaths, but in a secondary subgroup analysis, sevelamer treatment was associated with lower all-cause, but not cardiovascular, mortality rate for the patients older than 65 years $(P=0.02)$. The significance of this is unclear.

Block et al performed an analysis of the secondary end point of all-cause mortality from the 2005 study. ${ }^{45,64}$ This showed a significantly lower mortality rate in the sevelamer-treated group compared with calcium-based binder group $(P=0.016)$ and showed higher mortality rates in patients with higher coronary artery calcification scores. Neither the DCOR nor the Treat-toGoal study found significant differences in number of patients hospitalized or number of days in hospital. . $^{2763}$

Chiu et al analyzed the impact of pill burden on quality of life in 233 dialysis patients. The median daily pill burden was 19; in one-fourth of subjects, it exceeded 25 pills per day. Higher pill burden was independently associated with lower physical component summary scale scores on health-related quality of life (HR-QOL) on both univariate and multivariate analyses. Phosphate binders accounted for about one-half of the daily pill burden and $62 \%$ of the participants were nonadherent. There was only a modest relationship between pill burden from phosphate binders and adherence, and serum phosphorus levels. Perhaps unsurprisingly to many clinicians, no relationship was seen between adherence and serum phosphorus levels. Higher pill burden was clearly associated with lower HR-QOL, and increasing the number of prescribed pills did not seem to improve control and may come at the cost of poorer HR-QOL. ${ }^{64}$ In this regard, the reduced pill burden associated with use of the lanthanum carbonate may offer benefits to some patients. ${ }^{65}$

\section{Summary and conclusions}

The evidence supporting use of sevelamer in phosphate control rather than calcium-based binders is not clear, and there is limited data to support a difference. A 2007 systematic review found slight inferiority of sevelamer in terms of phosphate control, but this was associated with smaller increases in serum calcium and fewer episodes of hypercalcemia. ${ }^{66}$ Sevelamer may offer additional benefits in terms of its effects on cholesterol and uric acid levels and may play a role in the modulation of calcification, but as yet no prospective interventional study has clearly demonstrated this. A number of trials, including the Treatto-Goal study, have suggested attenuated vascular calcification with sevelamer compared with calcium-based binders, but this has not been replicated in other large multicentre trials, such as the BRiC or CARE-2 study. Interpretation of these findings is difficult, given the failure of multiple studies, to identify or control for other factors that might influence calcium load, such as type of calcium-based binder used, dialysate calcium levels, and the use of calcium supplements and vitamin D. In general, when correction for these factors is made, no significant difference has been reported. ${ }^{66}$ To date, there are no studies examining bone-related outcomes, such as fracture rate or 
bone pain. There is little evidence on the efficacy of sevelamer in reducing mortality and hospitalization. The only reports of decreased mortality rates come from secondary analyses alone. The balance of evidence, therefore, does not strongly support the use of sevelamer over the much less costly calcium-based binders except in patients at risk of hypercalcemic episodes. Further, research into cardiovascular and all-cause mortality over a longer time period would be needed to settle this issue.

Despite the relative paucity of data described above, sevelamer has established itself as the most widely used binder in the United States and the most widely used noncalcium-based binder worldwide. The link between calcium intake and the radiological appearance of vascular calcification in dialysis patients seems intuitive to many clinicians, and where sevelamer is affordable they choose to err on the side of caution, especially in the face of guidelines such as K/DOQI ${ }^{68}$ Affordability is a major issue for most health economies and in the light of recent economic events is likely to become more prominent. As pointed out by Isakova et a ${ }^{67}$ "No placebo-controlled studies have examined the impact of treatment with phosphorus binders on hard clinical end points, including survival. Indeed, although several studies aimed to determine which specific phosphorus binder is best for dialysis patients, there are no data on whether any phosphorus binder treatment vs none alters clinical outcomes". It is clearly a matter of great urgency that this deficiency in the dialysis knowledge base is rectified.

\section{Disclosure}

The authors report no conflicts of interest in this work.

\section{References}

1. Martin KJ, Gonzalez EA. Metabolic bone disease in chronic kidney disease. J Am Soc Nephrol. 2007;18:875-885.

2. Kidney Disease: Improving Global Outcomes (KDIGO) CKD-MBD Work Group. KDIGO clinical practice guideline for the diagnosis, evaluation, prevention, and treatment of chronic kidney disease-mineral and bone disorder (CKD-MBD). Kidney Int Suppl. 2009;(113):S1-S130.

3. Moe S, Drueke T, Cunningham J, et al. Definition, evaluation, and classification of renal osteodystrophy: a position statement from Kidney Disease: Improving Global Outcomes (KDIGO). Kidney Int. 2006;69:1945-1953.

4. Floege J, Ketteler M. Vascular calcification in patients with end-stage renal disease. Nephrol Dial Transplant. 2004;19 Suppl 5:V59-V66.

5. London GM, Pannier B, Guerin AP, Marchais SJ, Safar ME, Cuche JL. Cardiac hypertrophy, aortic compliance, peripheral resistance, and wave reflection in end-stage renal disease. Comparative effects of ACE inhibition and calcium channel blockade. Circulation. 1994;90: 2786-2796.

6. London GM, Marchais SJ, Guerin AP. Arterial stiffness and function in end-stage renal disease. Adv Chronic Kidney Dis. 2004;11:202-209.

7. Guerin AP, Pannier B, Marchais SJ, London GM. Cardiovascular disease in the dialysis population: prognostic significance of arterial disorders. Curr Opin Nephrol Hypertens. 2006;15:105-110.

8. Danisi G, van Os CH, Straub RW. Phosphate transport across brush border and basolateral membrane vesicles of small intestine. Prog Clin Biol Res. 1984;168:229-234.
9. Hutchison AJ. Oral phosphate binders. Kidney Int. 2009;75: 906-914.

10. Sherrard DJ, Andress DL. Aluminum-related osteodystrophy. Adv Intern Med. 1989;34:307-323.

11. Hutchison AJ, Gokal R. Towards tailored dialysis fluids in CAPD - the role of reduced calcium and magnesium in dialysis fluids. Perit Dial Int. 1992;12:199-203.

12. Hutchison AJ, Freemont AJ, Boulton HF, Gokal R. Low-calcium dialysis fluid and oral calcium carbonate in CAPD. A method of controlling hyperphosphatemia whilst minimizing aluminium exposure and hypercalcaemia. Nephrol Dial Transplant. 1992;7:1219-1225.

13. Goodman WG, Goldin J, Kuizon BD, et al. Coronary-artery calcification in young adults with end-stage renal disease who are undergoing dialysis. N Engl J Med. 2000;342:1478-1483.

14. Wilkes BM, Reiner D, Kern M, Burke S. Simultaneous lowering of serum phosphate and LDL-cholesterol by sevelamer hydrochloride (RenaGel) in dialysis patients. Clin Nephrol. 1998;50:381-386.

15. Barna MM, Kapoian T, O'Mara NB. Sevelamer carbonate. Ann Pharmacother. 2010;44(1):127-134.

16. Goldsmith DR, Scott LJ, Cvetkovic RS, Plosker GL. Sevelamer hydrochloride: a review of its use for hyperphosphatemia in patients with end-stage renal disease on haemodialysis. Drugs. 2008;68:85-104.

17. Burke S, Amin N, Incerti C, Plone M, Watson N. Sevelamer hydrochloride (Renagel), a nonabsorbed phosphate-binding polymer, does not interfere with digoxin or warfarin pharmacokinetics. J Clin Pharmacol. 2001;41:193-198.

18. Burke SK, Slatopolsky EA, Goldberg DI. RenaGel, a novel calciumand aluminium-free phosphate binder, inhibits phosphate absorption in normal volunteers. Nephrol Dial Transplant. 1997;12:1640-1644.

19. Wrong O, Harland C. Sevelamer and other anion-exchange resins in the prevention and treatment of hyperphosphatemia in chronic renal failure. Nephron Physiol. 2007;107:17-33.

20. Plone MA, Petersen JS, Rosenbaum DP, Burke SK. Sevelamer, a phosphate-binding polymer, is a non-absorbed compound. Clin Pharmacokinet. 2002;41:517-523.

21. Rosenbaum DP, Holmes-Farley SR, Mandeville WH, Pitruzzello M, Goldberg DI. Effect of RenaGel, a non-absorbable, cross-linked, polymeric phosphate binder, on urinary phosphorus excretion in rats. Nephrol Dial Transplant. 1997;12:961-964.

22. Genzyme Corporation. Renagel tablets (sevelamer hydrochloride) 400 and $800 \mathrm{mg}$. Highlights of prescribing information 2007. http:// www.renagel.com/docs/renagel_pi.pdf Accessed 8th July 2010.

23. Burke SK, Amin NS, Incerti C, Plone MA, Lee JW. Sevelamer hydrochloride (Renagel), a phosphate-binding polymer, does not alter the pharmacokinetics of two commonly used antihypertensives in healthy volunteers. J Clin Pharmacol. 2001;41:199-205.

24. Kays MB, Overholser BR, Mueller BA, Moe SM, Sowinski KM. Effects of sevelamer hydrochloride and calcium acetate on the oral bioavailability of ciprofloxacin. Am J Kidney Dis. 2003;42:1253-1259.

25. Pieper AK, Buhle F, Bauer S, et al. The effect of sevelamer on the pharmacokinetics of cyclosporin A and mycophenolate mofetil after renal transplantation. Nephrol Dial Transplant. 2004;19:2630-2633.

26. Merkle M, Wornle M, Rupprecht HD. The effect of sevelamer on tacrolimus target levels. Transplantation. 2005;80:707.

27. Chertow GM, Burke SK, Raggi P. Sevelamer attenuates the progression of coronary and aortic calcification in hemodialysis patients. Kidney Int. 2002;62:245-252.

28. Shaheen FA, Akeel NM, Badawi LS, Souqiyyeh MZ. Efficacy and safety of sevelamer. Comparison with calcium carbonate in the treatment of hyperphosphatemia in hemodialysis patients. Saudi Med J. 2004;25:785-791.

29. Asmus HG, Braun J, Krause R, et al. Two year comparison of sevelamer and calcium carbonate effects on cardiovascular calcification and bone density. Nephrol Dial Transplant. 2005;20:1653-1661.

30. Galassi A, Spiegel DM, Bellasi A, Block GA, Raggi P. Accelerated vascular calcification and relative hypoparathyroidism in incident haemodialysis diabetic patients receiving calcium binders. Nephrol Dial Transplant. 2006;21:3215-3222. 
31. Ferreira A, Frazao JM, Monier-Faugere MC, et al. Effects of sevelamer hydrochloride and calcium carbonate on renal osteodystrophy in hemodialysis patients. J Am Soc Nephrol. 2008;19:405-412.

32. Kokuho T, Toya Y, Kawaguchi Y, et al. Sevelamer hydrochloride improves hyperphosphatemia in hemodialysis patients with low bone turnover rate and low intact parathyroid hormone levels. Ther Apher Dial. 2007;11:442-448.

33. Takei T, Otsubo S, Uchida K, et al. Effects of sevelamer on the progression of vascular calcification in patients on chronic haemodialysis. Nephron Clin Pract. 2008;108:c278-c283.

34. Barreto DV, Barreto FC, de Carvalho AB, et al. Phosphate binder impact on bone remodeling and coronary calcification - results from the BRiC study. Nephron Clin Pract. 2008;110:c273-c283.

35. Brewster UC, Ciampi MA, Abu-Alfa AK, Reilly RF. Long-term comparison of sevelamer hydrochloride to calcium-containing phosphate binders. Nephrology (Carlton). 2006;11:142-146.

36. Qunibi WY, Hootkins RE, McDowell LL, et al. Treatment of hyperphosphatemia in hemodialysis patients: the Calcium Acetate Renagel Evaluation (CARE Study). Kidney Int. 2004;65:1914-1926.

37. Fournier A, Benyahia M, Claudia CP, Sadek T. Calcium on trial: beyond a reasonable doubt? Kidney Int. 2003;63:382-383.

38. Russo D, Miranda I, Ruocco C, et al. The progression of coronary artery calcification in predialysis patients on calcium carbonate or sevelamer. Kidney Int. 2007;72:1255-1261.

39. Ketteler M, Rix M, Fan S, et al. Efficacy and tolerability of sevelamer carbonate in hyperphosphatemic patients who have chronic kidney disease and are not on dialysis. Clin J Am Soc Nephrol. 2008;3:1125-1130.

40. Mehrotra R. Disordered mineral metabolism and vascular calcification in nondialyzed chronic kidney disease patients. J Ren Nutr. 2006;16:100-118.

41. Caglar K, Yilmaz MI, Saglam M, et al. Short-term treatment with sevelamer increases serum fetuin-a concentration and improves endothelial dysfunction in chronic kidney disease stage 4 patients. Clin J Am Soc Nephrol. 2008;3:61-68.

42. Othmane TH, Bakonyi G, Egresits J, et al. Effect of sevelamer on aortic pulse wave velocity in patients on hemodialysis: a prospective observational study. Hemodial Int. 2007;11 Supp1 3:S13-S21.

43. Sun PP, Perianayagam MC, Jaber BL. Sevelamer hydrochloride use and circulating endotoxin in hemodialysis patients: a pilot cross-sectional study. J Ren Nutr. 2009;19:432-438.

44. Moe SM, Chertow GM. The case against calcium-based phosphate binders. Clin J Am Soc Nephrol. 2006;1:697-703.

45. Block GA, Spiegel DM, Ehrlich J, et al. Effects of sevelamer and calcium on coronary artery calcification in patients new to hemodialysis. Kidney Int. 2005;68:1815-1824.

46. Friedman EA. Calcium-based phosphate binders are appropriate in chronic renal failure. Clin J Am Soc Nephrol. 2006;1:704-709.

47. Braun J, Asmus HG, Holzer H, et al. Long-term comparison of a calciumfree phosphate binder and calcium carbonate - phosphorus metabolism and cardiovascular calcification. Clin Nephrol. 2004;62:104-115.

48. Qunibi W, Muenz LR. Progression of calcification in the Calcium Acetate Renagel Evaluation-2 (CARE-2) Study. Am J Kidney Dis. 2008;52:1022-1023.

49. Garg JP, Chasan-Taber S, Blair A, et al. Effects of sevelamer and calcium-based phosphate binders on uric acid concentrations in patients undergoing hemodialysis: a randomized clinical trial. Arthritis Rheum. 2005;52:290-295.
50. Ohno I, Yamaguchi Y, Saikawa H, et al. Sevelamer decreases serum uric acid concentration through adsorption of uric acid in maintenance hemodialysis patients. Intern Med. 2009;48:415-420.

51. Gallieni M, Cozzolino M, Brancaccio D. Transient decrease of serum bicarbonate levels with sevelamer hydrochloride as the phosphate binder. Kidney Int. 2000;57:1776-1777.

52. Brezina B, Qunibi WY, Nolan CR. Acid loading during treatment with sevelamer hydrochloride: mechanisms and clinical implications. Kidney Int Suppl. 2004;(90):S39-S45.

53. Chertow GM, Burke SK, Dillon MA, Slatopolsky E. Long-term effects of sevelamer hydrochloride on the calcium x phosphate product and lipid profile of haemodialysis patients. Nephrol Dial Transplant. 1999;14:2907-2914.

54. Marco MP, Muray S, Betriu A, Craver L, Belart M, Fernandez E. Treatment with sevelamer decreases bicarbonate levels in hemodialysis patients. Nephron. 2002;92:499-500.

55. Sonikian MA, Pani IT, Iliopoulos AN, Koutala KG, Marioli SI, Vlassopoulos DA. Metabolic acidosis aggravation and hyperkaliemia in hemodialysis patients treated by sevelamer hydrochloride. Ren Fail. 2005;27:143-147.

56. Vlahakos DV, Retsa K, Kalogeropoulou S, Katsoudas S, Bacharaki D, Agroyannis B. Chronic acid-base perturbations in hemodialysis patients treated with sevelamer hydrochloride: a two-year follow-up study. Artif Organs. 2007;31:892-895.

57. Wrong OM, Harland CE. Sevelamer-induced acidosis. Kidney Int. 2005;67:776-777.

58. Harland C, Wrong O. Sevelamer 'carbonate': what's in a name? Nephrol Dial Transplant. 2010;25:1354-1355.

59. Sevelamer: constipation and occlusion. Complications, sometimes fatal. Prescrire Int. 2008;17:111.

60. Madan P, Bhayana S, Chandra P, Hughes JI. Lower gastrointestinal bleeding: association with sevelamer use. World J Gastroenterol. 2008; 14:2615-2616.

61. Fan S, Ross C, Mitra S, et al. A randomized, crossover design study of sevelamer carbonate powder and sevelamer hydrochloride tablets in chronic kidney disease patients on haemodialysis. Nephrol Dial Transplant. 2009;24:3794-3799.

62. Taylor MJ, Elgazzar HA, Chaplin S, Goldsmith D, Molony DA. An economic evaluation of sevelamer in patients new to dialysis. Curr Med Res Opin. 2008;24:601-608.

63. Suki WN. Effects of sevelamer and calcium-based phosphate binders on mortality in hemodialysis patients: results of a randomized clinical trial. J Ren Nutr. 2008;18:91-98.

64. Block GA, Raggi P, Bellasi A, Kooienga L, Spiegel DM. Mortality effect of coronary calcification and phosphate binder choice in incident hemodialysis patients. Kidney Int. 2007;71:438-441.

65. Hutchison AJ, Laville M. Switching to lanthanum carbonate monotherapy provides effective phosphate control with a low tablet burden. Nephrol Dial Transplant. 2008;23:3677-3684.

66. Tonelli M, Wiebe N, Culleton B, et al. Systematic review of the clinical efficacy and safety of sevelamer in dialysis patients. Nephrol Dial Transplant. 2007;22:2856-2866.

67. Isakova T, Gutierrez OM, Chang Y, et al. Phosphorus binders and survival on hemodialysis. J Am Soc Nephrol. 2009;20:388-396.

68. National Kidney Foundation (NKF) K/DOQI Guidelines. K/DOQI clinical practice guidelines for bone metabolism and disease in chronic kidney disease. Am J Kidney Dis. 2003;42:S7-S201.

Open Access Journal of Urology

\section{Publish your work in this journal}

The Open Access Journal of Urology is an international, peer-reviewed, open access journal publishing original research, reports, editorials, reviews and commentaries on all aspects of adult and pediatric urology in the clinic and laboratory including the following topics: Pathology, pathophysiology of urological disease; Investigation and treatment of

\section{Dovepress}

urological disease; Pharmacology of drugs used for the treatment of urological disease. The manuscript management system is completely online and includes a very quick and fair peer-review system, which is all easy to use. Visit http://www.dovepress.com/testimonials.php to read real quotes from published authors.

Submit your manuscript here: http://www.dovepress.com/open-access-journal-of-urology-journal 